\title{
Konflikte und maritime Sicherheitspolitik: Das Süd- chinesische Meer und die Transitrouten Südostasiens
}

\author{
Michael Fuker*
}

\begin{abstract}
The South China Sea is one of the most important und largest semi-enclosed seas in the world and a significant area of regional and international shipping lanes, particularly the Strait of Malacca. The area includes more than 200 islands, rocks and reefs and is rich in natural resources such as oil and natural gas, but also in living maritime resources. The islands are important for strategic and political reasons, especially since Asia's economy growth, and therefore the competing territorial claims over the South China Sea and its resources are numerous. In addition to traditional security threats the region and its maritime security is also plagued by non-traditional threats like piracy, maritime terrorism and illegal trade of arms, drugs and human beings.
\end{abstract}

Keywords: Territorial disputes, maritime security policy, piracy, maritime terrorism, rivalry over resources Grenzkonflikte, maritime Sicherheitspolitik, Piraterie, maritimer Terrorismus, Ressourcenwettbewerb

\section{Einleitung: Das Südchinesische Meer}

$\mathrm{D}$ as Südchinesische Meer ist neben der koreanischen Halbinsel und der Taiwan-Straße einer der drei Hauptschauplätze für potenzielle Auseinandersetzungen um nationale Interessen und die Bedrohung des Friedens und der Sicherheit in der Region Ostasien. Als eines der größten Meere weltweit beherbergt es eine Vielzahl von kleineren Inseln und Riffen und ist reich an lebenden und nicht-lebenden maritimen Ressourcen. Aufgrund der wichtigen strategischen Lage, bedingt durch zahlreiche Schiffsrouten, Handelswege und Ressourcen, wurde das Südchinesische Meer zu einem der wichtigsten Schauplätze des regionalen, aber auch des internationalen Wettbewerbs. Die kreuz und quer durch das Südchinesische Meer verlaufenden Schiffsrouten teilen Südostasien in eine maritime (Brunei, Indonesien, Malaysia, Philippinen und Singapur) und eine kontinentale Sphäre (Kambodscha, Laos, Myanmar, Thailand, Vietnam). ${ }^{1}$ Die angrenzenden Länder weisen eine Vielfalt von politischen Systemen auf, von kommunistischen bzw. sozialistischen Regimen in China und Vietnam über Demokratien in Malaysia, Singapur, Indonesien, den Philippinen bis hin zur absoluten Monarchie in Brunei. Die Schiffswege und Handelsrouten sind aber nicht nur für die Anrainerstaaten von wirtschaftlicher und strategischer Bedeutung, sondern auch für Japan, die USA und die europäischen Staaten. Der rasante wirtschaftliche Aufschwung Ostasiens hat weltweite wirtschaftliche und politische Anziehungskraft. Das Südchinesische Meer ist also ein für viele Staaten wichtiger, allerdings auch sehr unsicherer Raum. Territorialstreitigkeiten um Inseln an verschiedenen Stellen des Meeres - vor allem um bereits erkundete oder noch vermutete Rohstoffvorkommen in deren Umfeld - belasten die Beziehungen zwischen den Anrainerstaaten. Hinzu kommt, dass auch kriminelle Machenschaften wie Piraterie, Menschen, Drogen- und Waffenschmuggel im Südchinesischen Meer

* Michael Fuker ist Research Fellow am Austria Institut für Europa- und Sicherheitspolitik (AIES).

1 Shicun Wu/Keyuan Zou (2009): Maritime Security in the South China Sea. Regional Implications and International Cooperation, Ashgate Publishing London, S. 3-4. traditionell eine Rolle spielen. Auch Terrorismus ist eine permanente latente Bedrohung für die Seewege in der Region.

Als besonders verwundbar gilt die Straße von Malakka. Sie verbindet den Indischen Ozean mit dem Südchinesischen Meer und ist dadurch einer der wichtigsten Handels- und Transportwege weltweit. Circa 65.000 Schiffe passieren pro Jahr die Meerenge. Ihre Fracht macht etwa ein Drittel aller gehandelten Waren und die Hälfte der transportierten Energieträger aus. ${ }^{2}$

\section{Maritime Grenzkonflikte}

Die Grenzkonflikte im Südchinesischen Meer werden durch konkurrierende strategische und politische, aber auch durch wirtschaftliche Interessen geprägt. Die Interessenkonflikte beeinflussen auch die Beziehungen der Staaten untereinander. Insbesondere zwischen dem erstarkenden China und den Staaten der Association of Southeast Asian Nations (ASEAN) könnten neue Konflikte entstehen. Die Länder Südostasiens beobachten mit Sorge die maritime Aufrüstung der Großmacht China und fragen sich beunruhigt nach deren Motiven. Insbesondere fürchten sie, dass China künftig anders als bisher versucht sein könnte, Territorialkonflikte im Südchinesischen Meer zu seinen Gunsten militärisch zu lösen. ${ }^{3}$ Die zunehmende Modernisierung und Aufrüstung des chinesischen Militärs werden als wachsendes Interesse an regionaler militärischer Machtprojektion interpretiert. ${ }^{4}$

Permanenten Anlass für Streitigkeiten zwischen einigen ASEAN-Staaten untereinander sowie China bieten vor allem die wechselseitigen Besitzansprüche auf zwei kleinere Inselketten, die Paracels und die Spratlys. Diese zwei Inselketten sind nicht wegen ihrer Größe von strategischer Bedeutung, sondern aufgrund der erkundeten und vermuteten, jedoch noch nicht erschlossenen Öl- und Gasressourcen und der reichhaltigen

2 Stefan Mair (2010): Piraterie und maritime Sicherheit. Fallstudien zu Afrika Südostasien und Lateinamerika sowie Beiträge zu politischen, militärischen, rechtlichen und ökonomischen Aspekten, SWP-Studie, Berlin, S. 46.

3 Ralf Emmers (2009): The Changing Power Distribution in the South China Sea: Implications for Conflict Management and Avoidance. RSIS Working Paper, Rajaratnam School of International Studies, Singapore, S. 2

4 Bernt Berger (2010): Viel Lärm im maritimen Asien, SWP-Aktuell, Berlin, S. 4 
Fischvorkommen in den umliegenden Gewässern. Die Paracel-Inseln, welche vor der Zentralküste Vietnams und südöstlich der chinesischen Hainan-Insel liegen, werden sowohl von Vietnam als auch von China beansprucht. Im Jahr 1975 kam es erstmals zu Auseinandersetzungen zwischen beiden Staaten, als chinesische Streitkräfte, die People‘s Liberation Army (PLA), vietnamesische Truppen von den Paracel-Inseln vertrieben. Vietnam hat jedoch seither nicht aufgehört, den chinesischen Territorialanspruch zu bestreiten. Der Konflikt um die ParacelInseln ist auf beiden Seiten festgefahren, eine Lösung nicht in Sicht. ${ }^{5}$

Die Inselgruppe Spratly im südlichen Teil des Südchinesischen Meeres umfasst mehr als 170 geografische Formen, darunter auch Atolle und Riffe. Kaum 50 davon sind tatsächlich als Inseln zu bezeichnen. Insgesamt beanspruchen fünf Staaten (China, Taiwan, Vietnam, Philippinen und Malaysia) die Souveränität über diese Inselgruppe. Wie bei den Paracel-Inseln haben auch die Spratly-Inseln an sich keinen spezifischen wirtschaftlichen Eigenwert, aber die reichhaltigen Kohlenwasserstofflager unter dem Meeresboden und die Lage der Inseln nahe der vielbefahrenen Seeverbindungswegen, den sogenannten Sea Lines of Communication (SLOCs), fördert den Konkurrenzkampf um Ressourcen und strategische Ziele. Außer dem Sultanat Brunei, das für sich lediglich eine exklusive Wirtschaftszone (Exclusive Economic Zone - EEZ) fordert, haben bereits alle fünf genannten Staaten Teile der Inselgruppe besetzt und militärische Einheiten stationiert. ${ }^{6}$ Vietnam besetzt derzeit 25 Inseln und Riffe in den Spratlys und hat diese militärisch befestigt und gut ausgerüstet. Die Philippinen rangieren an zweiter Stelle bei der Anzahl der besetzten Gebiete. Sie kontrollieren gegenwärtig neun Teile der Gemeinde Kalayaan. Malaysia okkupiert derzeit fünf Gebiete, Taiwan hingegen die größte und zugleich am besten geschützte Insel der Spratlys, die Insel Itu-Aba. China selbst besetzt keine der Insel in den Spratlys, unterhält jedoch einige militärische Einrichtungen in ihrer Nähe. Die besetzenden Parteien sind in Sorge, dass eine chinesische Vormachtstellung auf der Inselgruppe dazu führen könnte, dass China seinen Einflussbereich im Herzen Südostasiens erweitert und zudem die angrenzenden Seeverbindungen kontrolliert. ${ }^{7}$ Diese Sorge teilen sie durchaus mit anderen Mächten, wie den USA und Japan, für welche die Transitrouten im Südchinesischen Meer von strategischer Bedeutung sind und die in Chinas Ambitionen eine Bedrohung eigener Interessen sehen.

Solange keine von allen Parteien anerkannte ostasiatische Sicherheitsarchitektur existiert besteht die Gefahr, dass konkurrierende Interessen einzelner Staaten alte Konflikte wieder neu aufbrechen lassen oder sich neue Spannungsfelder zwischen den Ländern entwickeln. ${ }^{8}$

5 Ian Storey/Carlyle A. Thayer (2010): The South China Sea Dispute: A Review of Developments and their Implications since the 2002 Declaration on the Conduct of Parties, in: Kesavan K.V./Singh, Daljit (2010): South and Southeast Asia. Responding to changing geo-political and security challenges, KW Publishers New Delhi, S. 57.

6 Ian Storey (2009): Maritime Security in Southeast Asia. Two Cheers for Regional Cooperation, in: Singh, Daljit (2009): Southeast Asian Affairs 2009, Institute of Southeast Asian Studies Singapore, S. 46-47.

7 Rommel C. Banlaoi (2010): Renewed Tensions and Continuing Maritime Security Dilemma in the South China Sea, National Defense College of Philippines, Philippines.

8 Bernt Berger (2010): Viel Lärm im maritimen Asien, op.cit., S. 4.
Der Konflikt um die Hoheit über die Paracel- und Spratly-Inseln zwischen den beteiligten Parteien hat historische und rechtliche Ursachen. Einerseits geht es um die Entdeckung und die frühere Besitznahme der Inseln. Andererseits spielt die wechselseitige Überlagerung von Seegrenzen und wirtschaftlichen Nutzungsrechten gemäß der Internationalen Seerechtskonvention der Vereinten Nationen eine Rolle. China betrachtet das Südchinesische Meer als ein exklusives chinesisches Meer und beansprucht aus historischen Gründen der Entdeckung und Besetzung fast das ganze Territorium. Die Aufrüstung der chinesischen Marine wird aus der Sicht anderer Anrainer nicht nur aufgrund möglicher Konflikte um die Souveränitätsrechte der umstrittenen Territorien forciert, sondern auch als Mittel angesehen, um die angrenzenden Seeverbindungen (Straße von Malakka, Singapur-Straße, Sundra-Straße und Lombok-Straße) unter Beijings Kontrolle zu bringen, die als Transitrouten für den wirtschaftlichen Reichtum und steigenden Energiebedarf Chinas eine wichtige Rolle spielen. ${ }^{9}$ Im Falle eines offenen Konflikts zwischen China und Taiwan oder einem anderen südostasiatischen Staat (z.B. Vietnam oder Philippinen) wären aus ihrer Sicht nur die US-amerikanischen Marineeinheiten imstande, den chinesischen Truppen zur See militärisch entgegenzutreten. Inwieweit die USA sich allerdings im zugespitzten Fall tatsächlich in die regionalen maritimen Territorialstreitigkeiten militärisch einmischen würden, ist schwer einzuschätzen. Die USA versuchen gegenwärtig offenbar eher den Eindruck bei ihrem wichtigsten wirtschaftlichen Partner und politischen Konkurrenten zu vermeiden, man wolle sich aus Eigennutz und um den eigenen Einfluss zu stärken in die regionalen Streitigkeiten einmischen. ${ }^{10}$ Washington konzentriert sich insofern momentan auf die Wahrung eigener Bewegungsspielräume für seine Pazifikflotte und auf die Gewährleistung einer freien Handelsschifffahrt im Raum Asien-Pazifik.

Die ASEAN-Mitgliedstaaten versuchen mit ihren machtpolitisch begrenzten Mitteln, ihr eigenes Modell der Konfliktvermeidung und Behandlung von Streitigkeiten in den Vordergrund zu rücken. Ihre unterschiedliche politische Verfasstheit und die bereits genannten Konflikte untereinander schränken das mögliche Spektrum gemeinsamer regionaler Sicherheitspolitik trotz erreichter Fortschritte in der Zusammenarbeit sichtlich ein. Die ASEAN setzt auf Kooperation und Soft Power. Hierdurch sollen weitere Spannungen mit Blick auf die strittigen Territorien und deren Ausweitung auf das gesamte Südchinesische Meer verhindert bzw. die Konflikte auf friedliche Art gelöst werden. Für das chinesische Konzept wirtschaftlicher Expansion ist dieses Herangehen durchaus förderlich. Mit der Unterzeichnung der Declaration on the Conduct of Parties in the South China Sea (DOC) im November 2002 und dem Treaty of Amity and Cooperation (TAC) im Oktober 2003 signalisierte China auch seine offizielle Unterstützung für das Konfliktmanagement-Modell der ASEAN. ${ }^{11}$ Die latenten Spannungen existieren jedoch fort und werden durch die diplomatischen Arrangements lediglich überlagert.

9 Rommel C. Banlaoi (2010): Renewed Tensions and Continuing Maritime Security Dilemma in the South China Sea, National Defense College of Philippines, Philippines, op. cit., S. 6.

10 Bernt Berger (2010): Viel Lärm im maritimen Asien, op. cit., S. 3

11 Ralf Emmers (2009): The Changing Power Distribution in the South China Sea, op. cit., S. 9 f. 
So gibt es seit 2007 erneut Spannungen um die Souveränität der Spratly-Inseln, auch weil die Entwicklung von zivilen und militärischen Einrichtungen auf allen besetzten Inseln, Riffen, etc. von den jeweiligen Ländern fortgesetzt wurde. Dies verdeutlicht, dass alle Anspruchsländer weiterhin bestrebt sind, auf den besetzten Territorien ihre Präsenz zu verstärken und ihr Anrecht auf Eigentum durchzusetzen. Die aufstrebende Seemacht China und die andauernde maritime Aufrüstung von Brunei, Malaysia, der Philippinen, Taiwan und Vietnam durch russische Mig-29-Kampfflugzeuge, englische Fregatten und schwedische U-Boote ist ein weiterer Indikator für die erhöhten Spannungen. Sie verschärfen das maritime Sicherheitsdilemma im Südchinesischen Meer. ${ }^{12}$

\section{Wettbewerb um Ressourcen}

Der Konkurrenzkampf um fossile Rohstoffe und Fischbestände nimmt aufgrund von wiederholten Engpässen bei Energieressourcen und angesichts des stetig wachsenden Bedarfs an Nahrungsmitteln zu. Die Interessen verschränken sich, wodurch Konflikte zusätzliche und einander wechselseitig beeinflussende Dimensionen erhalten. Der Territorialkonflikt um die Paracel-Inseln zwischen China und Indien wurde bereits 1974/1975 durch die damalige Ölkrise verstärkt. Heute verschärft ein sprunghaft ansteigender Energiebedarf bei gleichzeitiger rapider Kostensteigerung für die Erschließung und den Abbau nichterneuerbarer Energieträger den Wettbewerb und damit auch die Spannungen zwischen den Staaten. Langfristige Erschließungsperspektiven gewinnen in dem Maße an Gewicht, wie die Anzahl der noch nicht genutzten Reserven abnimmt. China und Vietnam sind im Wettlauf um die Ressourcen im Südchinesischen Meer nicht allein.

Regionale und externe Akteure, darunter auch Großmächte wie Indien, Japan und die USA, suchen auch außerhalb ihrer Hoheitsgewässer nach möglichen Öl- und Gasreserven im Meeresboden, um den eigenen zukünftigen nationalen Wohlstand zu gewährleisten und den Energiebedarf zu decken. In den Gewässern Südostasiens befindet sich eine Vielzahl sedimentärer Becken, die auf Öl- und Gasvorkommen hinweisen. Der erhöhte Energieverbrauch in den Ländern der Region und außerhalb lässt den Wettbewerb um Kohlenwasserstofflager wachsen. Wettbewerb kann Kooperationsinteressen beflügeln, aber auch durch egoistische Ausbeutung der Ressourcen zu neuen Spannungen führen. Immer mehr internationale Ölund Gaserkundungsindustrien siedeln sich in den Gewässern Südostasiens an, gewährleisten zwar die Energiesicherheit des jeweiligen Küstenstaats, aber zerstören dabei auch die maritime Umgebung. Die Region Südostasien ist derzeit mit ungefähr 37 aktiven und 135 geplanten Öl- und Gasplattformprojekten eines der aktivsten und konstruktivsten Energiegewinnungsgebiete weltweit. ${ }^{13}$ Nicht nur die einzelnen Länder stehen unter dem Druck, mehr Öl und Gas zu fördern, sondern auch große staatliche wie private Energiekonzerne. In den letzten Jahrzehnten sind vermehrt nationale Ölkonzerne (national oil companies - NOCs) gegründet worden, die den Wettbewerb

12 Rommel C. Banloi (2010): Renewed Tensions and Continuing Maritime Security Dilemma in the South China Sea, op. cit.

13 Sam Bateman/Joshua Ho/Jane Chan (2009): Good Order at Sea in Southeast Asia. RSIS Policy Paper, Rajaratnam School of International Studies, Singapore, S. 15. mit den internationalen Unternehmen (international companies - IOCs) aufnehmen. Zwischen ihnen herrscht aber nicht nur Wettbewerb. Vielmehr existieren auch internationale Ölfirmen (z.B. BP und ExxonMobil), die die nationalen Konzerne darin unterstützen, ihre Abhängigkeit von westlichen Energiegroßmächten zu verringern, indem sie modernere Technologien bereitstellen, Schulungen anbieten und seismische Aufschlüsse übermitteln. ${ }^{14}$

Nicht nur die Entdeckung und Erschließung von Öl- und Gasvorkommen stehen im Blickfeld vieler internationaler und regionaler Akteure, sondern auch die Versorgung mit Fisch und anderen lebenden maritimen Ressourcen, um die Nahrungssicherheit zu gewährleisten. Die Fischereiwirtschaft befindet sich weltweit in einer Krise und speziell Südostasien ist von der Erschöpfung der regionalen Fischvorkommen betroffen. Die Region ist nicht nur einer der größten Hersteller von Fischprodukten, sondern auch einer ihrer größten Konsumenten. Ähnlich wie bei den nichtlebenden Rohstoffen Öl und Gas nimmt der Konsum von Fischprodukten vor allem in den aufstrebenden Mächten zu. Ein Folgeproblem dieses Bedarfszuwachses ist die Zunahme der illegalen Fischerei. Der Wildwuchs der Fischwirtschaft und die damit einhergehende Vervielfachung der Anzahl von Fischerbooten auf der offenen See können durch nationale und internationale Institutionen nicht mehr kontrolliert werden, wodurch sich die sogenannte illegale, unregulierte und ungemeldete Fischerei (IUU Fishing) ungebremst weiter ausbreitet. Infolgedessen werden aber nicht nur die Fischbestände unkontrolliert dezimiert. Rücksichtsloses Handeln (Korallenfischerei, Einsatz von Explosivstoffen und Giften) zerstört die Umwelt und verletzt dadurch die wesentlichen ökologischen Interessen der Anrainer.

\section{Maritime Sicherheitspolitik}

\subsection{Aufstieg und Fall der Piraterie}

Seit den frühen 1990er Jahren lassen sich verstärkt Fälle von Piraterie im Südchinesischen Meer beobachten. Laut den Berichten des International Maritime Bureau (IMB) gab es im Zeitraum 1991 bis 2007 in der Straße von Malakka und im Südchinesischen Meer 963 Fälle von Piraterie. Die wesentlichen Faktoren, die zum Anstieg von Piratenüberfällen geführt haben, sind die einzigartige strategische Lage, die rasche Entwicklung der globalen Schiffsindustrie, die politische und wirtschaftliche Lage in den Küstenstaaten sowie die Schwierigkeiten, wirksame Anti-Piraterie-Maßnahmen zu vereinbaren. Vor allem dicht befahrene Schifffahrtswege wie die Straße von Malakka und auch das Südchinesische Meer sind gefährdet. Sie verbinden die westlichen und östlichen asiatischen Länder und sind zugleich wichtige Handelsrouten zwischen Ostasien, Afrika und Europa. ${ }^{15}$ In den meisten Fällen von Piraterie handelte es sich bisher um „Hit and run“-Raubüberfälle auf Fischerboote und kommerzielle Schiffe, also Überfälle, die meist nicht systematisch geplant sind, sondern zufällig verübt werden („spontane

14 Institute of Southeast Asian Studies (2009): Energy and Geopolitics in the South China Sea. Implications for ASEAN and its Dialogue Partners. ASEAN Studies Centre, Report No. 8, S. 22-23.

15 Shicun Wu/Keyuan Zou (2009): Maritime Security in the South China Sea, op. cit., S. 83ff. 
Piraterie“). ${ }^{16}$ Die Piraterie im heutigen Südostasien wurde durch ein Zusammenspiel politischer und wirtschaftlicher Faktoren, unter anderem durch die Finanz- und Wirtschaftskrisen, begünstigt. Der steigende Seeverkehr in der Straße von Malakka und im Südchinesischen Meer führte dabei nicht nur zu einem Navigationsstau in den Seestraßen, sondern hatte auch negative Auswirkungen auf die lokale maritime Umgebung und deren lebende Ressourcen, insbesondere die Fischbestände. Piraterie ist ein transnationales Verbrechen, von dem oft verschiedene Parteien aus mehreren Ländern profitieren. Zusätzlich zur „spontanen Piraterie“ existieren auch kriminelle Syndikate. Sie bilden Netzwerke, die zumeist neben der Piraterie auch in anderen organisierten kriminellen Aktivitäten wie etwa Schmuggel, Menschen- und Waffenhandel aktiv sind. Im Gegensatz zu den kleinen Piratengruppen verfügen diese Syndikate über hochmoderne Waffen und erhebliche Ressourcen, die sie befähigen, selbst mittelgroße Schiffe zu entführen, die gestohlene Fracht zu veräußern und eine Neuregistrierung von gestohlenen Schiffen zu veranlassen. Diese werden inzwischen als sogenannte "phantom ships “ bezeichnet. ${ }^{17}$

In den südostasiatischen Ländern wurde die Piraterie viele Jahre lang nicht ernst genommen bzw. ignoriert, da andere nationale und transnationale Sicherheitsbedrohungen wie Terrorismus, illegale Fischerei oder Menschen-, Waffen- und Drogenhandel im Zentrum der Aufmerksamkeit standen. Erst verstärkter Druck durch Staaten, deren Wirtschaft besonders stark von sicheren Seeverbindungen abhängt, veranlasste Anrainerstaaten der Straße von Malakka (Indonesien, Malaysia, Singapur) die Bekämpfung der Piraterie zu einer Priorität ihrer Sicherheitspolitik zu machen. Initiativen zur Bekämpfung der Piraterie weisen seit 2004 unilaterale, bilaterale und trilaterale Komponenten auf, die zum Teil aber auch noch von externen Akteuren abhängig sind. ${ }^{18}$ Mit dem 2004 beschlossenen MALSINDO (Malaysia, Singapur, Indonesia)-Programm zur Durchführung koordinierter Patrouillen in der Straße von Malakka wurde zum einen die Einmischung internationaler Akteure in die regionale Sicherheitspolitik größtenteils unterbunden und zum anderen das ASEAN-typische Nichteinmischungsprinzip in innerstaatliche Angelegenheiten auch auf maritimer Ebene umgesetzt. Zwei Jahre später wurde das MALSINDO-Programm in Malacca Strait Patrols (MSP) umbenannt. Das Programm umfasst folgende Elemente: (1) die Kontrolle der Seegebiete (Malacca Straits Surface Patrols), (2) das Luftraumüberwachungsprogramm („eyes in the sky“ - EIS) und (3) den Austausch von nachrichtendienstlichen Erkenntnissen (Intelligence Exchange Group- IEG). ${ }^{19}$

Trotz vermehrter bilateraler/multilateraler Kooperationen und Initiativen ist die Problematik der Piraterie noch zu wenig auf regionaler Ebene, speziell in der ASEAN, verankert. Da nicht alle ASEAN-Mitgliedstaaten direkt von der Piraterie betroffen sind, gibt es bisher auch keine einheitliche Strategie zu ihrer Bekämpfung, sondern lediglich verschiedene allgemeine Erklärungen und Abkommen zu transnationaler Kriminalität und

16 Stefan Mair (2010): Piraterie und maritime Sicherheit, op. cit.; vgl. auch: Marco Bünte (2009): Piraterie in Südostasien - Neuere Entwicklungen und Perspektiven. GIGA Journal of Current Southeast Asian Affairs 28, S. 90.

17 Shicun Wu/Keyuan Zou (2009): Maritime Security in the South China Sea, op. cit., S. 90.

18 Stefan Mair (2010): Piraterie und maritime Sicherheit, op. cit., S. 48-49. 19 Ebd. S. 50.
Terrorismus. Andere an der Sicherheit der Seeverbindungen interessierte Staaten haben sich hingegen auf konkrete Maßnahmen geeinigt. So unterstützen beispielsweise die USA und Japan die Einrichtung und den Betrieb regionaler Satellitenüberwachungs- und Kommunikationssysteme. Mit dem 2006 ratifizierten Regional Cooperation Agreement on Combating Piracy and Armed Robbery against Ships in Asia (ReCAAP) wurde die erste zwischenstaatliche Vereinbarung über eine multilaterale Zusammenarbeit zur Bekämpfung der Piraterie ins Leben gerufen. Mit Hilfe dieser Initiative und der operativen Plattform des ReCAAP-Informationstauschzentrums sollen das Kommunikationsniveau weiter verbessert und spezielle „Capacity building“-Maßnahmen durchgeführt werden. Die maritime Sicherheitssituation in Südostasien hat sich seit 2004 unter anderem auch durch diese regionalen Kooperationen und die Unterstützung externer Akteure verbessert; die Zahl der Angriffe durch Piraten ist deutlich zurückgegangen. Allerdings darf die Bedrohung durch Piraterie in der Region weder als obsolet erachtet noch unterschätzt werden. Zudem werden die Erfolge der bisherigen Zusammenarbeit nur dann Bestand haben, wenn die Kooperation weiter vertieft wird und sich weitere Staaten daran beteiligen. Die bereits erwähnte Malacca Straits Patrols Initiative bietet hier eine ausbaufähige Grundlage. Die regionalen Küstenstaaten sollten sich aber nicht nur primär auf die Bekämpfung der Piraterie konzentrieren, sondern zusätzliche Ressourcen für die Bearbeitung von deren Ursachen aufwenden. Die Verbesserung der sozioökonomischen Bedingungen in den Anrainergesellschaften und die Stärkung von Prinzipien guter Regierungsführung (good governance) würde das Entstehen von Piraterie deutlich senken. ${ }^{20}$

Aus den bisher schon erfolgreichen regionalen Kooperationen und Aktivitäten zur Bekämpfung der Piraterie im Südchinesischen Meer und in der Straße von Malakka könnten so auch Lehren für andere Seegebiete der Welt (z.B. die Küste von Somalia) gezogen werden.

\subsection{Maritimer Terrorismus}

Eine zusätzliche und andere Art von Gefahr für den Handel und die maritimen Ressourcen in der Region ist der sogenannte maritime Terrorismus. Piraterie und Terrorismus sind nicht deckungsgleich, weder in Bezug auf die jeweiligen Ursachen noch auf die Formen und Strategien des Gewalteinsatzes. Piraten und Schmuggler verfolgen durchweg das Ziel des ökonomischen Profits und unterscheiden sich dadurch von zumeist politisch motivierten Terroristen. Eine trennscharfe Abgrenzung beider Aktionsfelder ist gleichwohl zunehmend schwierig, da selbsternannte terroristische Gruppen sich auch durch organisierte Kriminalität finanzieren, wie umgekehrt Organisationen mit einem kriminellen Hintergrund ihre Verbrechen durch politische Forderungen oder Begründungen zu tarnen suchen. Eine besondere Gefahr durch Terrorismus droht den großen Seehäfen.

Im Zeitalter der Globalisierung entwickeln sich diese Seehäfen von traditionellen Durchlässen zwischen Meer und Land zu Anbietern vollständiger Logistiknetzwerke. Die Entwicklung des Containerverkehrs hat diesen Trend noch beschleunigt.

20 Ian Storey (2009): Maritime Security in Southeast Asia, op. cit., S. 45. 
Seit den Anschlägen vom 11. September 2001 in den USA und dem Attentat von Bali im Jahre 2002 ist die potenzielle Gefährdung auch des maritimen Raumes durch Terrorangriffe verstärkt ins Zentrum sicherheitspolitischer Debatten gerückt. In den Gewässern Südostasiens gibt es bisher faktisch allerdings nur wenige Belege einer wachsenden Bedrohung der Häfen durch terroristische Anschläge. ${ }^{21}$ Im Jahr 2004 sorgten zwei Fälle von maritimem Terrorismus für Aufregung: im Februar 2004 ein Bombenattentat auf eine philippinische Fähre, bei dem 116 Passagiere ums Leben kamen, und im Juni desselben Jahres die Entführung eines Öltankers am nördlichen Eingang der Straße von Malakka. Ungeachtet dessen, dass es bisher keine weiteren größeren Anschläge oder Schiffsentführungen gegeben hat, befürchten Politiker und Sicherheitsexperten in der Region aber dennoch, dass Terroristen künftig versucht sein könnten und auch in der Lage wären, z.B. in Zusammenarbeit mit Piratengruppen, terroristische Anschläge im Südchinesischen Meer durchzuführen. Im Einzelnen werden vor allem die beiden folgenden Szenarien für möglich erachtet: ${ }^{22}$

- Selbstmordattentate auf Handels- und Militärschiffe sowie in Häfen nach dem Muster der Angriffe auf den US-Zerstörer USS-Cole im Jahr 2000 im Hafen von Aden und der Zerstörung des französischen Öltankers Limburg 2002 in jemenitischen Gewässern;

- die Entführung von Schiffen, um Waffen und Sprengstoff zu schmuggeln, Lösegeld für Geiseln zu erpressen oder einen terroristischen Angriff auf ungeschützte Schiffe oder Häfen durchzuführen.

Campbell und Gunaratna ${ }^{23}$ weisen außerdem auf drei weitere mögliche Szenarien maritimer terroristischer Anschläge hin: (1) Schiffe könnten als Mittel eingesetzt werden, um terroristische Anschläge durchzuführen. (2) Ein Schiff mit hochexplosivem Material an Bord könnte als Waffe gegen Häfen oder andere Schiffe eingesetzt werden. (3) Schiffe könnten selbst das Ziel von terroristischen Anschlägen sein, um an Bord befindliche Urlauber oder Reisende zu treffen.

Eine spezielle maritime Terrorabwehrstrategie für Südostasien existiert bisher nicht. Die ASEAN hat im Jahre 2001 immerhin einen gemeinsamen Aktionsplan ${ }^{24}$ verabschiedet, mit dem die Mitgliedstaaten versuchen wollen, das Risiko von Terroranschlägen zu reduzieren:

- vermehrter Informationsaustausch zwischen den Mitgliedsländern bezüglich terroristischer Aktivitäten;

- Stärkung und Erweiterung der bestehenden Kooperationen zwischen der ASEAN Ministers Meeting on Transnational Crime (AMMTC) und anderen relevanten Einrichtungen der ASEAN, die sich mit der Bekämpfung und Verhinderung terroristischer Aktivitäten auseinandersetzen;

- Weiterentwicklung von regionalen Ausbildungsprogrammen zur Unterstützung der vorhandenen Fähigkeiten der

21 Marco Bünte (2009): Piraterie in Südostasien - Neuere Entwicklungen und Perspektiven, op. cit., S. 93.

22 Graham Gerard Ong (2004): Ships can be dangerous too: Coupling Piracy and Maritime Terrorism in Southeast Asia's Maritime Security Framework. ISEAS Working Paper No.1, Institute of Southeast Asian Studies, Singapore, S. 7-8.

23 Tanner Campbell/Rohan Gunaratna (2003): Maritime Terrorism, Piracy and Crime, in: Rohan Gunaratna: Terrorism in the Asia-Pacific: Threat and Response, Eastern Universities Press Singapore, S. 80-83.

24 http://www.aseansec.org/12636.htm (Abfrage: 13.1.2011).
ASEAN-Mitgliedstaaten, terroristische Aktivitäten besser zu erkennen, zu beobachten und zu bekämpfen;

- stärkere Einbeziehung von internationalen Akteuren in übergreifende Foren wie das ASEAN-Regionalforum (ARF) und die sogenannte ASEAN+3 (die zehn Mitglieder der ASEAN plus Japan, Südkorea und China), um neue Ideen und unterschiedliche Maßnahmen der Terrorismusbekämpfung zu diskutieren.

Die ASEAN-Mitgliedstaaten und ihre Kooperationspartner tun sich jedoch weiterhin schwer, verbindliche Absprachen untereinander hinsichtlich praktischer Maßnahmen zu treffen.

\section{Fazit und Ausblick}

Das Südchinesische Meer wird auch in Zukunft im Fokus internationaler Sicherheitspolitik bleiben. Erstens werden dort vermehrt regionale und internationale Akteure nach Öl- und Gasvorkommen suchen und diese erschließen, um die jeweiligen Bedürfnisse hinsichtlich der Energieversorgung sicherzustellen. Nicht nur die Energie- sondern auch die Nahrungssicherheit ist bei einigen regionalen und internationalen Staaten, speziell China und Indien, zunehmend gefährdet, wodurch sich das Interesse an der Ausbeutung der Ressourcen des Südchinesischen Meeres noch verstärken wird. Die Gefahr der Überfischung ist akut gegeben, verstärkt noch durch illegale Fischerei und steigende Nachfrage der benachbarten bevölkerungsreichsten Länder der Welt. Unklar sind die möglichen politischen und speziell sicherheitspolitischen Folgen dieser Entwicklungen, nicht zuletzt im Kontext der weiter schwelenden Territorialkonflikte in der Region. Der größte Risikofaktor ist China. Sollte China künftig seine territorialen Ansprüche im Südchinesischen Meer militärisch lösen wollen, würde dies gewiss nicht nur die Region in einen Konfliktherd verwandeln, sondern auch entferntere Akteure auf den Plan rufen, deren Interessen dann bedroht wären. Ob und wie die USA in einer solchen Situation gegensteuern könnten, ist ebenso unklar wie die Frage, ob ASEANs Politik der Kooperation und Ausbalancierung von Interessen imstande wäre, eine solche Entwicklung zu verhindern.

Schließlich bleiben die Bekämpfung von Piraterie und die Gefahr des maritimen Terrorismus in der Region auf Dauer ein Thema und Anlass zur Sorge. Durch die erhöhten Anstrengungen auf Seiten regionaler Staaten und die Unterstützung Japans, Chinas, Indiens und den USA konnte eine Ausbreitung der Piraterie in den Küstenwässern erfolgreich unterbunden werden. Um diese Entwicklung fortzusetzen, müssen aber nicht nur die laufenden Anstrengungen aufrechterhalten werden, sondern auch neue regionale und internationale Kooperationen entstehen. Als Folge der Wirtschaftskrise 2008/2009 wird gegenwärtig vor allem in den ärmeren und militärisch schwächeren Ländern der Region mit einem erneuten Anstieg der Piraterie gerechnet. Da diese Staaten einerseits besonders anfällig für die Ansiedelung von Piratennetzwerken sind, sie andererseits aber auch die hohen Kosten der Bekämpfung der Piraterie nicht allein tragen können, sind sie auf verstärkte Hilfe von außen angewiesen. Die Stabilisierung der Lage im Südchinesischen Meer und in den angrenzenden Gewässern liegt insofern im globalen Interesse. 